\title{
Entacapone prolongs levodopa response in a one month double blind study in parkinsonian patients with levodopa related fluctuations
}

\author{
H M Ruottinen, U K Rinne
}

\begin{abstract}
Objectives-To establish, in a double blind manner, the antiparkinsonian effects of repeated dosing with entacapone, a peripheral COMT inhibitor. Methods-A one month, cross over study was conducted. During the two four-week treatment periods, entacapone $(200 \mathrm{mg})$ or placebo was given with each levodopa dose four to 10 times daily. Motor responses were repeatedly quantified using the motor part of UPDRS. Plasma levodopa and its metabolites were measured.
\end{abstract}

Results-Entacapone prolonged the availability of levodopa in the plasma and thus to the brain by decreasing its peripheral O-methylation and slowing its elimination rate, without affecting the maximum plasma levodopa concentration or the time to maximum concentration. Corresponding with the pharmacokinetic findings, entacapone prolonged the duration of motor response to an individual levodopa/DDC inhibitor dose by 34 minutes $(24 \%, P=0.001)$ and dyskinesiae by 39 minutes $(37 \%, P=0.002)$ compared with placebo, without affecting their magnitude or starting time. Entacapone treatment resulted in a reduction of $16 \%$ in the mean total daily levodopa dose due to dyskinesiae. Also, according to the home diaries, the mean daily "on" time increased by $2 \cdot 1$ hours compared with placebo, despite the lowered mean levodopa intake.

Conclusion-The efficacy of repeated entacapone dosing as an adjuvant to levodopa/DDC inhibitor treatment for Parkinson's disease with levodopa related fluctuations is verified.

$(千$ Neurol Neurosurg Psychiatry 1996;60:36-40)

Keywords: entacapone; catechol- $O$-methyltransferase inhibitor; levodopa; Parkinson's disease with levodopa related fluctuations

Department of Neurology, University of Turku, Turku, Finland

H M Ruottinen U K Rinne

Correspondence to: Dr H M Ruottinen, Department of Neurology, University of Turku, FIN 20520 Turku, Finland.

Received 11 July 1995 and in revised form and in revised form

Accepted 1 September 1995

Entacapone is a peripherally acting, selective, and reversible second generation catechol-Omethyltransferase (COMT) inhibitor, ${ }^{12}$ which has been studied as an adjuvant to levodopa/dopadecarboxylase (DDC) inhibitor treatment mainly in advanced Parkinson's disease with motor fluctuations. Peripheral COMT inhibition with entacapone has improved the bioavailability of levodopa ${ }^{3-7}$ and prolonged the antiparkinsonian response to levodopa. ${ }^{5-8}$ So far, only open ${ }^{45}$ or single dose, double blind ${ }^{68}$ studies have been reported on entacapone.

The aim of this study was to evaluate in a double blind manner the antiparkinsonian effects of entacapone found in the earlier open studies. For this purpose we conducted a one month, double blind study to evaluate the effects of repeated entacapone dosing on the pharmacokinetics and metabolism of levodopa and on motor responses to levodopa in patients with Parkinson's disease with end of dose fluctuations

\section{Methods}

SUBJECTS

Twenty six patients with idiopathic Parkinson's disease and levodopa related fluctuations in disability were studied. Twenty three patients completed the study; three patients discontinued because of unexpected adverse events or intercurrent disease and were excluded from the efficacy analyses. The mean age of the patients included in the efficacy analyses was $61 \cdot 3$ (SD 7.9) years, ranging from 46 to 75 years. Their "on" phase modified Hoehn and Yahr stage ${ }^{910}$ was mostly grade 3 (grade $2, \mathrm{n}=7$; grade $2 \cdot 5, \mathrm{n}=1$; grade $3, \mathrm{n}=14$; grade $4, \mathrm{n}=1$ ). The mean duration of Parkinson's disease was 14 (SD 5), range 7-25 years, the duration of levodopa treatment was 11 (SD 5) years, and the duration of fluctuations in disability ranged from three to 19 years. All patients were receiving levodopa and four patients were taking amantadine, seven were taking anticholinergic drugs, 11 were taking dopamine agonists, and seven were taking selegiline concomitantly. Only patients without psychiatric or severe physical illness were included in the study, which was conducted according to the principles of the Declaration of Helsinki. The study was approved by the Joint Commission on Ethics of Turku University and the Turku University Central Hospital. All subjects gave signed informed consent after written and verbal information about the study.

\section{STUDY DESIGN}

The study followed a double blind, randomised, placebo controlled, comparative, cross over design with two four-week treatpatients had been on stable standard levodopa/DDC inhibitor (either benserazide ( $n$ $=20)$ or carbidopa $(n=3)$ medication for at ment periods without a wash out period. The 
least two weeks before the study. During the two treatment periods, $200 \mathrm{mg}$ of entacapone ((E)-2-cyano- $N, N$-diethyl-3-(3,4-dihydroxy-5nitrophenyl)propenamide, OR-611, OrionPharma International, Espoo, Finland) or placebo in a randomised order were given four to 10 times daily with the patients' optimal, individual levodopa/DDC inhibitor treatment. Thus, the daily entacapone dose varied from 800 to $2000 \mathrm{mg}$ depending on the patients' individual number of daily levodopa doses. The study consisted of three test days, called levodopa test days: one at the beginning of the study (control day) and one test day at the end of each four-week treatment period. After the control day the patients were randomised. For the second treatment period the study medication was switched. On the three test days the pharmacokinetics and metabolism of levodopa and the patients' motor response to levodopa/ DDC inhibitor were determined. During the two treatment periods, the amount of a single levodopa dose and the frequency of levodopa dosing could be adjusted according to the clinical response or side effects induced by levodopa. However, the levodopa test was performed on each test day with the individual, constant morning levodopa dose throughout the study. The dosage of other Parkinson's disease medication was kept unchanged.

On the levodopa test days, as inpatients in the Department of Neurology, the patients were given a single oral dose of entacapone $(200 \mathrm{mg}$ ) or placebo (except on the control day) along with their individual levodopa/ DDC inhibitor dose at $800 \mathrm{am}$ when the morning dose of other parkinsonian medication or any other possible medication was also given. Apart from the variation in entacapone/placebo dosing, the three levodopa test days were otherwise identical. The levodopa test days were preceded by an overnight fast and at least six hours without Parkinson's disease medication. On all test days the patients were on a low protein diet and received breakfast at $1030 \mathrm{am}$ and lunch at $1230 \mathrm{pm}$.

Venous blood samples for the measurement of plasma concentrations of levodopa and its main metabolites were collected before the intake of any study drugs (0-sample) and every 30 minutes thereafter until 1000 am and, after that, every 60 minutes for at least four hours or for as long as the clinical response continued. The samples were treated as described previously ${ }^{3}$ and protected from light during the handling and storing procedures. An ion pair reversed phase high pressure liquid chromatography (RP-HPLC) method with coulometric detection ${ }^{11}$ was used for determinations of levodopa, 3-O-methyldopa (3-OMD), 3,4dihydroxyphenyl acetic acid (DOPAC), and homovanillic acid (HVA).

The motor response to levodopa was assessed using the motor part of the unified Parkinson's disease rating scale (UPDRS) ${ }^{10}$ supplemented with scoring of dyskinesiae (the levodopa test). ${ }^{12}$ The average of two scorings performed half an hour apart before the intake of the study drug was used as a baseline value. Thereafter, the scorings were performed every
30 minutes, by the same person (HMR) on each test day.

\section{PHARMACOKINETIC ANALYSIS}

The maximum plasma concentration $\left(\mathrm{C}_{\max }\right)$, the time of maximum concentration $\left(t_{\max }\right)$, and the terminal elimination half life $\left(t_{t e l}\right)$ of levodopa were determined according to standard methods as described previously. ${ }^{7}$ The area under the plasma concentration time curve (AUC) was calculated by the linear trapezoidal method ${ }^{13}$ from time zero to four hours $\left(\mathrm{AUC}_{0-4 \mathrm{~h}}\right)$ for levodopa and 3-OMD; and from time zero to the last detectable concentration $\left(\mathrm{AUC}_{0 \text {-lddc }}\right)$ for DOPAC and HVA. Values below the limit of determination $(20 \mathrm{ng} / \mathrm{ml})$ were included as zero in the pharmacokinetic analysis.

\section{CLINICAL RESPONSE}

The primary clinical efficacy variable was the duration of motor response to levodopa (the "on" time) in the levodopa test assessed with the motor part of UPDRS after levodopa/ DDC inhibitor alone (control day), and after placebo and entacapone medication. The secondary efficacy variables were the magnitude of motor response (maximal change from baseline in the total motor score) during the levodopa test, the starting time of the motor response (onset latency), the time to reach the lowest score during the levodopa test (peak latency), and the duration, magnitude (maximum score), and starting time of dyskinesiae. Each item of the UPDRS and dyskinesiae was scored $^{10}$ and the responses were defined as described earlier. ${ }^{7}$ The clinical response to levodopa was also evaluated by home diaries, which the patients filled in during the three preceding days before each levodopa test day for recording the daily "on" time and frequency of dyskinesiae. Averages over three home diary days were calculated and compared between treatments, using changes from control day as a secondary efficacy variable.

\section{SAFETY}

Adverse experiences were recorded by active inquiry during the test days and control visits in each treatment period. Blood samples for determination of haematological and clinical chemistry variables and urine samples were taken as precautionary measures before the patients entered the study and at the end of the two treatment periods. A 12 lead ECG was recorded before the patients entered the study, repeatedly during the test days, and in the morning after the last test day (poststudy day). Blood pressure and heart rate (supine and standing) were measured both before the initiation of the study, repeatedly during the clinical scoring on the test days, on the control visits, and on the poststudy day.

\section{STATISTICAL METHODS}

The statistical analyses of clinical, pharmacokinetic, and haemodynamic variables were done with analysis of variance (ANOVA) for cross over design. ${ }^{14}$ For the $t_{\max }$ of levodopa, a non-parametric Friedman's test was used. 
Orthogonal contrasts were used to evaluate differences between the placebo and entacapone treatments. The $95 \%$ confidence intervals $(95 \%$ CIs) of the mean differences were calculated. The changes from control day were calculated for placebo and entacapone test days, and thereafter these changes were compared. In statistical comparisons a two way risk level of $5 \%$ was used as the limit of significance.

\section{Results}

PHARMACOKINETIC VARIABLES OF LEVODOPA AND ITS METABOLITES

Figure 1 shows the effects of entacapone and placebo on the plasma levodopa concentrations. The mean changes from control day in the $\mathrm{AUC}_{0} \mathrm{Hh}_{\mathrm{h}}$ of levodopa differed between the treatments. The $\mathrm{AUC}_{0-4 \mathrm{~h}}$ of levodopa increased significantly by $35 \%$ after entacapone medication compared with placebo $(\mathrm{P}$ $<0.0001)$. Also the $t_{\mathrm{tel}}$ of levodopa differed between the entacapone and placebo medication. Entacapone prolonged the $t_{\mathrm{icl}}$ of levodopa significantly by $32 \% \quad(20 \mathrm{~min})$ compared with placebo $(\mathrm{P}<0.0001)$. There was no statistically significant change in the mean $\mathrm{C}_{\max }$ and $\mathrm{t}_{\max }$ of levodopa after entacapone medication $(\mathrm{P}=0.4$ and $\mathrm{P}=0.6$ respectively)

The $\mathrm{AUC}_{0 \text { th }}$ of 3-OMD, the COMT dependent metabolite of levodopa, decreased significantly by $63 \%$ after the four-week entacapone treatment compared with placebo $(\mathrm{P}<0.0001)$. The $\mathrm{AUC}_{0 \text {-tlde }}$ of DOPAC increased significantly by $201 \%(\mathrm{P}<0.0001)$ and the $\mathrm{AUC}_{0 \text {-lddc }}$ of HVA decreased by $17 \%(\mathrm{P}$ $=0.04)$ after entacapone compared with placebo.

CLINICAL RESPONSE

Figures 2 and 3 show the mean total motor scores and the mean duration of motor response to levodopa during the levodopa test. The table presents the mean changes from control day in the motor response to levodopa and in the dyskinesiae on placebo and on entacapone medication. The mean duration of motor response to individual levodopa dose on the control day was 146 (SD 63) minutes,

Figure 1 Mean plasma concentrations of levodopa during the levodopa test after an individual oral dose of levodopa/DDC inhibitor alone (control day), and after four weeks of concomitant placebo or entacapone in parkinsonian patients (mean (SEM), $n=23$ ).

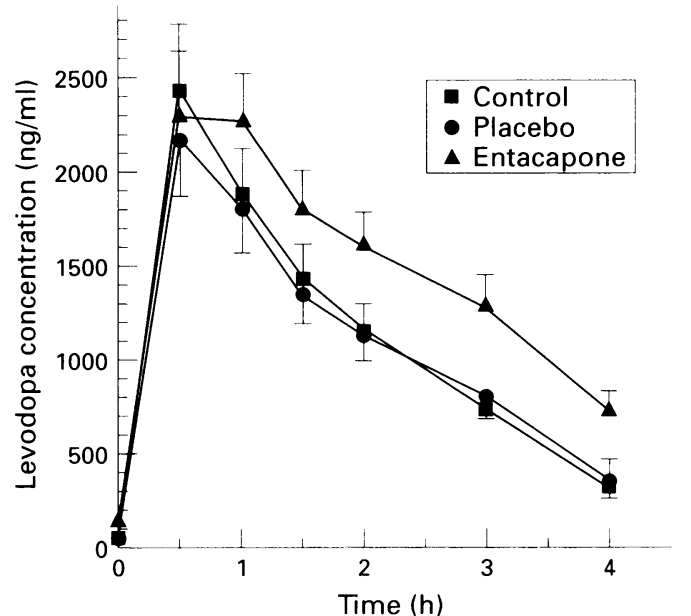

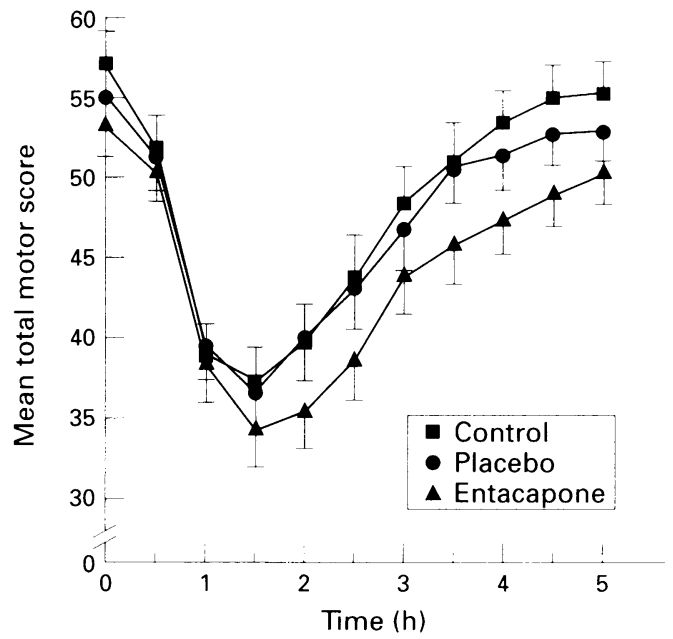

Figure 2 Mean total motor scores during the levodopa test after levodopa/DDC inhibitor alone (control day), after four weeks of concomitant administration of placebo or entacapone in parkinsonian patients (mean (SEM), $n=23$ ).

ranging from 30 to 240 minutes. The mean changes from control day in the duration of motor response differed significantly between the treatments. Entacapone increased the mean "on" time significantly by $24 \%, 34 \mathrm{~min}$ utes, compared with placebo, with $95 \% \mathrm{CI}$ of 16-54 minutes $(P=0.001)$. The mean onset latency, peak latency, and magnitude of motor response to levodopa remained virtully unaffected after entacapone medication compared with placebo $(P=0.9, P=0.8$, and $P=0.6$ respectively). All the patients except one had dyskinesiae during the levodopa tests. The mean duration of dyskinesiae on the patients' individual levodopa dose was 119 (SD 74) minutes, ranging from 0 to 240 minutes. Entacapone lengthened the duration of dyskinesiae significantly by $37 \%$ (39 $\mathrm{min} ; 95 \% \mathrm{CI}$ 17-62 min, $\mathrm{P}=0.002$ ) compared with placebo. The onset of dyskinesiae was slightly delayed by $19 \%(10 \mathrm{~min})$ and the magnitude of dyskinesiae slightly increased, by $16 \%$, after entacapone medication but they did not reach

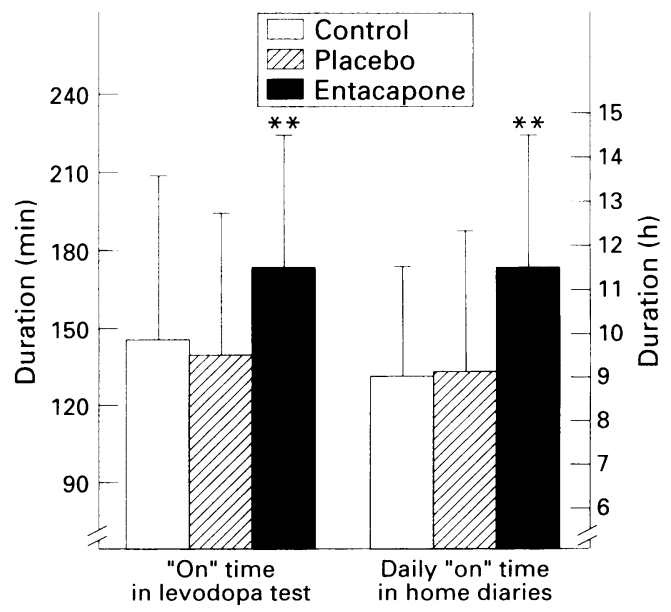

Figure 3 Mean duration of motor response ("on" time, min) during the levodopa test, and daily total "on" time according to patients' subjective assessment described in home diaries on their individual levodopa/DDC inhibitor treatment alone (control day), or on concomitant placebo or entacapone medication (mean (SD); $n=23$ during the levodopa test; $n=21$, except on placebo $(n=22)$ in home diaries $)$ 
Mean changes from control day in the motor response to levodopa and in the dyskinesiae on placebo and entacapone medication in parkinsonian patients

\begin{tabular}{|c|c|c|c|c|c|c|}
\hline \multirow[b]{2}{*}{ Treatment } & \multicolumn{3}{|c|}{ Motor response } & \multicolumn{3}{|l|}{ Dyskinesiae } \\
\hline & $\begin{array}{l}\text { Change in } \\
\text { duration } \\
\text { (min) }\end{array}$ & $\begin{array}{l}\text { Change in } \\
\text { onset latency } \\
\text { (min) }\end{array}$ & $\begin{array}{l}\text { Change in } \\
\text { magnitude }\end{array}$ & $\begin{array}{l}\text { Change in } \\
\text { duration } \\
\text { (min) }\end{array}$ & $\begin{array}{l}\text { Change in } \\
\text { onset of } \\
\text { dyskinesiae } \\
\text { (min) }\end{array}$ & $\begin{array}{l}\text { Change in } \\
\text { magnitude }\end{array}$ \\
\hline $\begin{array}{l}\text { Placebo } \\
\text { Entacapone }\end{array}$ & $\begin{array}{l}-7(50) \\
27(55)^{\star \star}\end{array}$ & $\begin{array}{l}4(19) \\
4(23)\end{array}$ & $\begin{array}{l}-2(4) \\
-1(5)\end{array}$ & $\begin{array}{r}-13(58) \\
26(55)^{\star}\end{array}$ & $\begin{array}{r}4(42) \\
\times 14(32)\end{array}$ & $\begin{array}{l}0.3(2.4) \\
1.3(2.8)\end{array}$ \\
\hline
\end{tabular}

Results are mean $(\mathrm{SD})$

statistical significance $(P=0 \cdot 1$ and $P=0 \cdot 2$ respectively).

During the four-week entacapone treatment the mean total daily dose of levodopa was reduced by $16 \%$, from 860 (SD 320$) \mathrm{mg}$ to 720 (SD 250) $\mathrm{mg}(\mathrm{P}=0.001)$, because of dyskinesiae. In 11 patients out of 23 the single levodopa dose was lowered, and in three of them also the frequency of dosing was reduced.

According to the patients' subjective assessment, described in the home diaries, there was a significant difference between the treatments. The mean daily total "on" time on the control day was 9.0 (SD 2.5 ) hours, ranging from $5 \cdot 1$ to 13.9 hours. On entacapone medication it increased by 2.5 (SD 2.3) hours, and on placebo by $0 \cdot 4(\mathrm{SD} 2 \cdot 5)$ hours (fig 3$)$. Thus the mean daily "on" time increased significantly by $23 \%(2 \cdot 1$ hours; $95 \%$ CI $0 \cdot 7-3 \cdot 5$ hours, $P=0.005$ ), during entacapone treatment compared with placebo. The mean frequency of dyskinesiae, reported by the patients in the home diaries, did not differ significantly between the treatments $(P=0 \cdot 8)$.

There were 58 newly occurring adverse experiences on entacapone and 39 on placebo medication. These were mainly mild or moderate and the most frequent during the four-week entacapone treatment (number of patients on entacapone/placebo) were diarrhoea or loose stools $(4 / 1)$, abdominal pain or discomfort (5/3), confusion (2/0), anxiety and insomnia (2/0), faintness (3/1), mood elevation $(2 / 0)$, and increased dyskinesiae (6/1). The mean supine and standing systolic and diastolic blood pressure decreased during the levodopa tests. The difference in supine and standing systolic blood pressure one hour after drug intake increased slightly more after entacapone than with placebo $(P=0.05)$. However, the mean maximal changes in the supine or standing systolic or diastolic blood pressure values measured during the levodopa tests did not differ between entacapone and placebo treatmant. Orthostatic hypotension occurred during the levodopa tests in five patients on the control day, in six patients on entacapone treatment, and in four patients on placebo medication. The heart rate and ECG findings were not influenced by entacapone.

Three patients discontinued the study due to unexpected adverse events or intercurrent disease. In one patient with nausea, vomiting, and abdominal pain on placebo medication, gastric ulcer was later diagnosed. Another patient, on heavy diuretic treatment for oedema, had orthostatic hypotension on enta- capone medication and this continued on placebo medication. The study was discontinued due to syncope and hyponatraemia. A third patient discontinued the study due to severe nosebleed during entacapone.

\section{Discussion}

This four-week, cross over study evaluated in a double blind manner the efficacy of entacapone as an adjuvant to levodopa therapy. The earlier studies with repeated entacapone dosing have been open labelled. Furthermore, the therapeutic efficacy of entacapone was monitored using the motor part of UPDRS which is a comprehensive method for quantitating the clinical response. In principle, the present results are in accordance with the findings obtained in the previous studies carried out with entacapone in parkinsonian patients.

Entacapone slowed the elimination of levodopa from plasma and increased its $\mathrm{AUC}_{0-4 \mathrm{~h}}$ without affecting its $\mathrm{C}_{\max }$ or $\mathrm{t}_{\max }$. The high 3OMD concentrations were effectively lowered by the four-week entacapone treatment. The $\mathrm{AUC}_{0-4 \mathrm{~h}}$ of 3-OMD decreased also in those 12 patients whose daily levodopa intake was not reduced. Thus, the decrease in the 3-OMD concentration was mainly due to inhibition of $O$-methylation, and not to lowered levodopa dose alone, as also reported by Nutt et al. ${ }^{5} \mathrm{~A}$ wash out period between the treatments was not applied in this study due to the short half life of entacapone ( 1.4 to 3.6 hours).$^{12}$ Despite the long half life of 3-OMD (15 to 18 hours), ${ }^{15}$ its concentration is assumed to be normalised after four days - that is, after five times its half life. Thus, presumably, no carry over effect interfered with the scorings at the end of the next four-week treatment period. The increased $\mathrm{AUC}_{0 \text {-ldd }}$ of DOPAC, a monoamine oxidase dependent metabolite of dopamine, presumably reflects the shifting of dopamine metabolism from the $O$-methylation to oxidation in the periphery, and is also associated with the decreased COMT-dependent metabolism of DOPAC to HVA. The decreased $\mathrm{AUC}_{0 \text {-tldc }}$ of HVA, the end product of dopamine metabolism, is assumed to reflect improved peripheral COMT inhibition.

In accord with the pharmacokinetic results, entacapone caused a both statistically and clinically significant 34 minutes of increase in duration of motor response to each levodopa/DDC inhibitor dose without affecting the magnitude, onset, or peak latency of the motor response. Correspondingly, entacapone prolonged the duration of dyskinesiae but their magnitude and starting time remained virtually unaffected. The present increase in "on" time was equal to that after a $200 \mathrm{mg}$ dose of entacapone in the earlier double blind, single dose studies ${ }^{6}{ }^{8}\left(34 \mathrm{~min} v 35 \mathrm{~min}^{8}\right.$ and $33 \mathrm{~min}^{6}$ ) but lower compared with the "on" time obtained in open studies ( $34 \mathrm{~min} v$ about 1 hour $^{5}$ ). The prolonged motor response to each levodopa/DDC inhibitor dose was reflected as a clinically significant $2 \cdot 1$ hours of increase in the total daily "on" time, as reported by the patients in their home diaries. 
Thus home diaries are a useful tool for assessing therapeutic response in parkinsonian patients with end of dose fluctuations. The use of entacapone resulted in a reduction in the requirement of the total daily levodopa dose and, thereby, it might alleviate the possible long term complications of levodopa treatment in Parkinson's disease.

The present double blind study showed that entacapone prolonged the availability of levodopa in plasma and thus to the brain by inhibiting the peripheral $O$-methylation of levodopa and slowing its elimination rate. Entacapone also caused a clinically significant increase in "on" time, despite the lowered mean levodopa intake. For the first time the findings of earlier open studies with repeated entacapone dosing were confirmed in a double blind manner. The efficacy of the peripheral COMT inhibitor entacapone as an adjuvant to levodopa/DDC inhibitor treatment of Parkinson's disease with levodopa related fluctuations is thus verified.

We are grateful to Orion-Pharma, especially to Dr Ariel Gordin, for the generous supply of entacapone. This study was supported by grants from the Medical Research Council of the Academy of Finland. We gratefully acknowledge P Lönnberg and $P$ Marjamäki for their assistance during the study; $M$. Karlsson and $T$ Wikberg for the bioanalytical determinations; T Kyyrä for statistical advice, and M Mäkimartti for advice on pharmacokinetic methodology.

1 Männistö PT, Tuomainen P, Tuominen RK. Different in vivo properties of three new inhibitors of catechol $O$ methyltransferase in the rat. $B r f$ Pharmacol 1992;105: 569-74.

2 Nissinen E, Linden IB, Schultz E, Pohto P. Biochemical and pharmacological properties of a peripherally acting
catechol-O-methyltransferase inhibitor Entacapone. Naunyn Schmiedeberg's Arch Pharmacol 1992;346:262-6.

3 Myllylä VV, Sotaniemi KA, Illi A, Suominen K, Keränen T. Effect of entacapone, a COMT inhibitor, on the pharmacokinetics of levodopa and on cardiovascular responses in patients with Parkinson's disease. Eur f Clin Pharmacol 1993;45:419-23.

4 Kaakkola S, Teräväinen H, Ahtila S, Rita H, Gordin A. Effect of entacapone, a COMT inhibitor, on clinical disability and levodopa metabolism in parkinsonian patients. Neurology 1994;44:77-80.

5 Nutt JG, Woodward WR, Beckner RM, et al. Effect of peripheral catechol-O-methyltransferase inhibition on the pharmacokinetics and pharmacodynamics of levodop in parkinsonian patients. Neurology 1994;44:913-9.

6 Ruottinen H, Rinne UK. A dose-finding clinical and pharmacokinetic study of entacapone as an adjuvant to levodopa treatment in Parkinson's disease. Neurology 1994; 44(suppl 2):A258.

7 Ruottinen HM, Rinne UK. Effect of one month's treatment with peripherally-acting COMT inhibitor, entacapone, on pharmacokinetics and motor response to levodopa in advanced parkinsonian patients. Clinical Neuropharmacol 1996(in press).

8 Merello M, Lees AJ, Webster R, Bovingdon M, Gordin A. Effect of entacapone, a peripherally acting catechol- $O$ methyltransferase inhibitor, on the motor response to acute treatment with levodopa in patients with Parkinson's disease. $\mathcal{F}$ Neurol Neurosurg Psychiatry 1994; 57:186-9.

9 Hoehn MM, Yahr MD. Parkinsonism: onset, progression, and mortality. Neurology 1967;17:427-42.

10 Fahn S, Elton RL, Members of the UPDRS Development Committee. Unified Parkinson's Disease Rating Scale. In: Fahn S, Marsden CD, Goldstein M, Calne DB, eds. In: Fahn S, Marsden CD, Goldstein M, Calne DB, eds.
Recent developments in Parkinson's disease Vol II. Florham Recent developments in Parkinson's disease Vol II. Florham Park, N

11 Wikberg T. Simultaneous determination of levodopa, its main metabolites and carbidopa in plasma by liquid chromatography. F Pharm Biomed Anal 1991;9:167-76.

12 Esteguy M, Bonnet AM, Kefalos J, Lhermitte F, Agid Y. Le test a la L-dopa dans la maladie de Parkinson. Rev Neurol 1985;141:413-5.

13 Gibaldi M, Perrier D. Pharmacokinetics. 2nd ed. New York: M Dekker, 1982

14 Jones B, Kenward M. Design and analysis of cross-over trials. London: Chapman and Hall, 1989.

15 Kuruma I, Bartholini G, Tissot R, Pletscher A. The metabolism of L-3-O-methyldopa, a precursor of dopa in man. Clin Pharmacol Ther 1971;12:678-82.

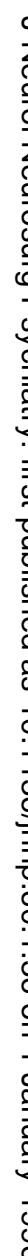

